\title{
Synthesis, Spectral, and Biological Properties of Copper(II) Complexes of Thiosemicarbazones of Schiff Bases Derived from 4-Aminoantipyrine and Aromatic Aldehydes
}

\author{
Ram K. Agarwal, ${ }^{1}$ Lakshman Singh, ${ }^{2}$ and Deepak Kumar Sharma ${ }^{2}$ \\ ${ }^{1}$ Department of Chemistry, School of Pure and Applied Sciences, University of the South Pacific, \\ PO Box 1168, Suva, Fiji Islands \\ ${ }^{2}$ Department of Chemistry, Lajpat Rai Postgraduate College, Sahibabad 201005 (Ghaziahad), India
}

Received 9 February 2005; Revised 15 April 2005; Accepted 25 April 2005

\begin{abstract}
We have synthesized a novel series of Schiff bases by condensation of 4-aminoantipyrine and various aromatic aldehydes followed by reaction with thiosemicarbazide. These thiosemicarbazones are potential ligands toward transition metal ions. The reaction of copper(II) salts with 4 [N-(benzalidene)amino] antipyrinethiosemicarbazone (BAAPTS), $4\left[\mathrm{~N}\right.$-( $4^{\prime}$-methoxybenzalidene) amino] antipyrinethiosemicarbozone (MBAAPTS), 4[N-(4'-dimethylamino benzalidene) amino] antipyrinethiosemicarbazone (DABAAPTS), and 4[N-(cinnamalidene) amino] antipyrinethiosemicarbazone (CAAPTS) resulted in the formation of solid complexes with the general composition $\mathrm{CuX}_{2} \cdot\left(\mathrm{H}_{2} \mathrm{O}\right)(\mathrm{L})\left(\mathrm{X}=\mathrm{Cl}, \mathrm{Br}, \mathrm{NO}_{3}, \mathrm{NCS}\right.$, or $\mathrm{CH}_{3} \mathrm{COO} ; \mathrm{L}=\mathrm{BAAPTS}$, MBAAPTS, DABAAPTS, or CAAPTS). These complexes were characterized through elemental analysis, molecular weight, electrical conductance, infrared, electronic spectra, and magnetic susceptibilities at room temperature. Copper(II) complexes with BAAPTS and MBAAPTS were screened for antibacterial and antifungal properties and have exhibited potential activity. Thermal stabilities of two representative complexes were also investigated.
\end{abstract}

Copyright (c) 2006 Ram K. Agarwal et al. This is an open access article distributed under the Creative Commons Attribution License, which permits unrestricted use, distribution, and reproduction in any medium, provided the original work is properly cited.

\section{INTRODUCTION}

Thiosemicarbazones are now well established as an important class of sulfur donor ligands particularly for transition metal ions [1-3]. This is due to remarkable biological activities observed for these compounds, which has since been shown to be related to their metal complexing ability. These compounds present a great variety of biological activity ranging from antitumour, fungicide, bactereocide, antiinflammatory, and antiviral activities [4-8]. We have previously examined the chelating behaviour of some NNS donor thiosemicarbazones having pyrazolone ring in several metal complexes with the object of gaining more information about their nature of coordination and related structural and spectral properties $[9,10]$.

In the present work, we report the synthesis, magnetospectral, antibacterial, and antifungal properties of copper(II) complexes of $4[\mathrm{~N}$-(benzalidene) amino] antipyrinethiosemicarbazone (BAAPTS), $4\left[\mathrm{~N}\right.$-( $4^{\prime}$-methoxybenzalidene) amino] antipyrinethiosemicarbazone (MBAAPTS), $4[\mathrm{~N}-$ (4'-dimethylaminobenzalidene) amino] antipyrinethiosem- icarbazone (DABAAPTS) and 4[N-(cinnamalidene) amino] antipyrinethiosemicarbazone (CAAPTS) (Figure 1).

\section{EXPERIMENTAL}

\section{Materials}

Copper(II) salts, for example, copper(II) chloride, bromide, nitrate, or acetate, were obtained from BDH. AR-grade and were used as such. $\mathrm{Cu}(\mathrm{SCN})_{2}$ was prepared by mixing copper chloride (in ethanol) and ethanolic solution of potassium thiocyanate in $1: 2$ molar ratio. Precipitated $\mathrm{KCl}$ was filtered off, and the filtrate having copper(II) thiocyanate was used immediately for complex formation. All the four thiosemicarbazones were prepared in the laboratory by reported procedure [11]. All solvents obtained commercially were distilled before use.

The antibacterial activities of both thiosemicarbazones, that is, BAAPTS and MBAAPTS complexes of copper(II), were studied by the usual cup-plate-agar-diffusion method $[12,13]$. The compounds were screened for their antibacterial activity against the following microorganisms: (a) gram 


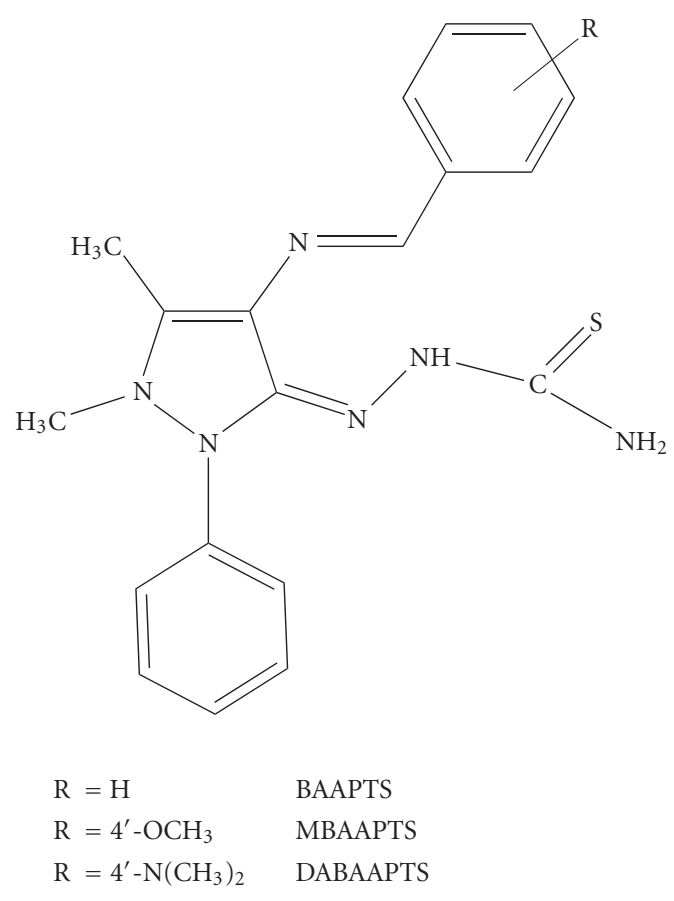

(a)

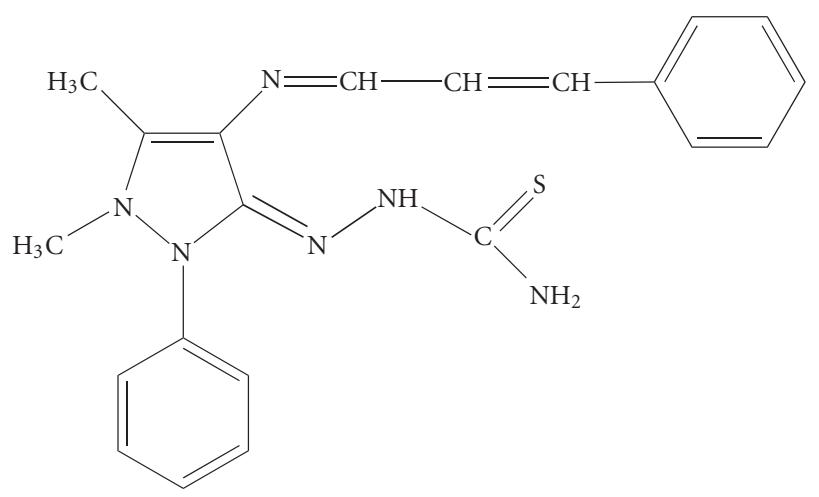

CAAPTS

(b)

FIGURE 1: Structures of different thiosemicarbazones.

positive staphylococcus aureus ( $S$ aureus), (b) gram negative $E$ coli. The cup-plate-agar-diffusion method comprises of the following steps.

(1) Preparation of media, sterilization, and tubing.

(2) Sterilization of the cleaned glass apparatus.

(3) Pouring of the seeded medium into sterilized petridishes and cutting of the cups.

(4) Pouring of the dilute solution of the compounds into the tubs.

(5) Incubation at a particular temperature.

(6) Determination of the "zones of inhibition."
The composition of the test media is the factor, which often exerts the greatest effect upon the drug activity. This is particularly true for thiosemicarbazones, since inhibitors of these compounds appear to be present in the common bacteriological culture medium. Efficient media of known chemical composition are available for many species such as $S$ aureus and $E$ coli. In addition to the composition of the test media, its $\mathrm{pH}$ is a factor which may directly or indirectly influence the activity of a drug. The $\mathrm{pH}$ of the test media taken for $S$ aureus and $E$ coli was adjusted in the range $7.6 \pm 0.1$. The composition of the basal media used in the experiments was (i) sodium chloride $=6.0 \mathrm{gm}$, (ii) peptone $=10.0 \mathrm{gm}$, (iii) beef extract $=3.0 \mathrm{gm}$, (iv) yeast extract $=2.0 \mathrm{gm},(\mathrm{v})$ sucrose $=1.5 \mathrm{gm}$, (vi) agar-agar $=3.0 \%$, and (vii) distilled water $=1.0$ litre.

\section{Procedure}

The measured quantity of the culture of the test organism $(0.5 \mathrm{~mL})$ was added to each heated (nearly $\sim 55^{\circ} \mathrm{C}$ ) agarmedia tubes. The tubes were shaken well, and the inoculated media were poured on to the sterilized petridishes and then allowed to set in a refrigerator maintained at $4-8^{\circ} \mathrm{C}$. The test solutions of $500 \mu \mathrm{g} / \mathrm{mL}$ and $1000 \mu \mathrm{g} / \mathrm{mL}$ dilutions of the respective thiosemicarbazones were prepared in a mixture of DMF and $\mathrm{H}_{2} \mathrm{O}(3: 7, \mathrm{v} / \mathrm{v})$. Five cups of $5 \mathrm{~mm}$ diameter were cut in the culture media on the pertidishes. A compound solution of particular dilution $(500 \mu \mathrm{g} / \mathrm{mL}$ or $1000 \mu \mathrm{g} / \mathrm{mL})$ was put in the outer four cups of one of the petridishes, and the second solution was put in the four cups of other petridishes. The central cups of all the petridishes were filled with the controlled solution, and all the petridishes were allowed to remain in the refrigerator maintained at $\sim 10^{\circ} \mathrm{C}$ for $\sim 1 \mathrm{hr}$ to allow diffusion of the solution. The petridishes were then transferred to an incubator maintained at $\sim 35^{\circ} \mathrm{C}$ and kept for nearly $30 \mathrm{hrs}$. The zones of inhibition formed were measured with calipers. The control of DMF and $\mathrm{H}_{2} \mathrm{O}(3: 7, \mathrm{v} / \mathrm{v})$ showed no activity. The activity of the compounds are represented by size of the diameter in $\mathrm{mm}$. The antifungal activity of the compounds was screened by using filter paper disc diffusion method. The tests were carried out by taking $6 \mathrm{~mm}$ diameter filter paper discs against the fungi ( $A$ niger and $C$ albicans).

\section{Synthesis of the complexes}

All the copper(II) complexes were synthesized by following general procedure. The corresponding copper(II) salt and the appropriate thiosemicarbazone in equimolar ratio were dissolved, separated in ethanol, and mixed together. The reaction mixture was boiled under refluxing state for $\sim 4 \mathrm{hrs}$. On cooling at room temperature, a microcrystalline complex was separated. It was filtered under suction, and the crystals were washed with cold ethanol and finally with anhydrous diethyl ether and kept in a desiccator over fused $\mathrm{CaCl}_{2}$.

\section{Analyses}

Copper contents of the complexes were estimated complexometrically with EDTA using murexide and erichrome black 
$\mathrm{T}$ as an indicator after decomposing the complexes with concentrated $\mathrm{H}_{2} \mathrm{SO}_{4}$ and $\mathrm{H}_{2} \mathrm{O}_{2}$ [14]. The halogens were estimated by Volhard's method [15]. The thiocyanate was estimated by titrating slightly acidic solution of the complex with standard silver nitrate solution. Sulfur was estimated gravimetrically as $\mathrm{BaSO}_{4}$. The percentage of nitrogen was determined by Kjeldahl method. The molecular weight of the complexes was determined in the laboratory cryoscopically in freezing nitrobenzene using a Beckmann thermometer of accuracy $\pm 0.01^{\circ} \mathrm{C}$. The conductivity measurements were carried out using a Toshniwal conductivity bridge type CL 01/01 and dip type cell operated at 220 volts, AC mains. All the measurements were done at room temperature in $\mathrm{PhNO}_{2}$. The magnetic measurements on powder form of the complexes were carried out at room temperature on Gouy's balance using anhydrous copper sulfate as calibrant. The infrared spectra of the complexes were recorded on a Perkin Elmer Infrared Spectrophotometer model-521 in KBr/CsI in the range of $4000-200 \mathrm{~cm}^{-1}$ at the University of Delhi, Delhi, India. Diffused reflectance spectra of the solid compounds were recorded on a Beckmann-DK-2A Spectrophotometer at the University of Delhi. Thermogravimeteric analysis of the complexes was carried out in static air with open sample holder and a small platinum boat, the heating rate was $6^{\circ} \mathrm{C} / \mathrm{min}$.

\section{RESULTS AND DISCUSSION}

The reaction of $\mathrm{Cu}^{2+}$ salts with BAAPTS, MBAAPTS, DABAAPTS, and CAAPTS gave complexes of the general composition $\mathrm{CuX}_{2}(\mathrm{~L})\left(\mathrm{H}_{2} \mathrm{O}\right)\left(\mathrm{X}=\mathrm{Cl}, \mathrm{Br}, \mathrm{NO}_{3}, \mathrm{NCS}\right.$, or $\mathrm{CH}_{3} \mathrm{COO} ; \mathrm{L}=$ BAAPTS, MBAAPTS, DABAAPTS, or CAAPTS). The analytical data of these complexes are presented in Table 1. All the complexes are quite stable and could be stored for months without any appreciable change. The complexes do not have sharp melting point, but decomposed on heating beyond $250^{\circ} \mathrm{C}$. These complexes are generally soluble in common organic solvents. The molar conductance values of the complexes in $\mathrm{PhNO}_{2}$ are presented in Table 1. The values are too low to account for any dissociation, therefore the complexes are considered to be nonelectrolytes [16]. The molecular weights determined by cryoscopic method in $\mathrm{PhNO}_{2}$ (Table 1) are in broad agreement with the conductance data. The observed magnetic moments of all these complexes (Table 1) are in 1.81-1.92 BM range. The observed magnetic moments of the complexes are consistent with the presence of a single unpaired electron $[17,18]$.

\section{Biological properties}

A number of authors [19-23] were interested to investigate the biological and medicinal properties of transition metal complexes of thiosemicarbazones. Thomas and Parmeswaran [20] studied the antitumour activities of $\mathrm{Mn}^{2+}$, $\mathrm{Co}^{2+}, \mathrm{Ni}^{2+}$, and $\mathrm{Cu}^{2+}$ chelates of anthracene-9-carboxaldehyde thiosemicarbazone. Murthy and Dharmaraja [21] reported the cytotoxic activity of phenylglyoxal bis(thiosemicarbazone) against Ehrlich ascites carcinoma cells. These compounds were also screened for antimicrobial activity on $B$ subtilis and $E$ coli. They inhibited the bacterial growth considerably. Garg et al [24] have recently reported the antifungal activity of some transition metal complexes of 2-(2'hydroxybenzylidene)aminophenyl benzimidazole. All complexes were screened against Alternaria alternata and Aspergillus niger by spore germination inhibition method at concentrations 100, 500, and 1000 ppm using Dithane M45 as a standard. Recently, Singh [25] published a review article on metal complexes of glutathione and their biological properties. Copper(II) is the most important oxidation state of copper in many physiological systems. $\mathrm{Cu}$ (II) complexes of glutathione were tested for their antifungal activity against some plant pathogenic fungi using slide germination technique [26]. $\mathrm{Cu}(\mathrm{I})$ is another important oxidation state of copper in physiological systems. $\mathrm{Cu}(\mathrm{I})$-thioamino complex formation serves not only to improve the chelation therapy for treating intoxication, but may also provide a better understanding of many facets of normal copper metabolism [27-29], since copper is an essential trace metal which can adopt a redox system in biological system allowing it to play a pivotal role in physiology. Copper homeostasis in biological system is well characterized, involving several proteins such as glutathione, metallothione, ATPase, Menkes, and Wilso proteins as well as the cytoplasmic copper chaperons. In view of the biological relevance of copper(II) complexes, in the present studies, the antibacterial activities of the copper(II) complexes of BAAPTS and MBAAPTS and standard drugs (ampicillin and teracycline) were screened by the agar-cup method in DMF solvent at a concentration of $50 \mu \mathrm{g} / \mathrm{mL}$ and were checked against gram positive bacteria $B$ subtilis and $S$ aureus and gram negative bacteria $E$ coli and $S$ typhi (Table 2). Diameters of zone of inhibition (in $\mathrm{mm}$ ) of standard drug ampicillin against gram positive bacteria $B$ subtilis and $S$ aureus and gram negative bacteria $E$ coli and $S$ typhi were found to be $15,13,17$, and 18 , respectively, while tetracycline gave $18,17,21$, and 22 , respectively, under identical conditions, Table 2 show that all copper(II)-thiosemicarbazone complexes have moderate antibacterial activities against these bacteria. Both thiosemicarbazones and their copper(II) complexes were screened for their antifungal activities against two fungi (A niger and $C$ albicans). The results (Table 2) showed that almost all complexes showed nearly the same extent of activity, but they are less active compared to salicylic acid. It is interesting to note that due to the presence of methoxy group and comparatively faster diffusion of MBAAPTS complexes, they showed increased activity than that of BAAPTS complexes. These compounds were found to be efficient antifungal agents.

\section{INFRARED}

A study and comparison of infrared spectra of free ligands (BAAPTS, MBAAPTS, DABAAPTS, or CAAPTS) and their $\mathrm{Cu}^{2+}$ complexes (Tables 3 and 4 ) imply that these ligands behave as neutral tridentate and the copper(II) is coordinated through $\mathrm{N} \& \mathrm{~N}$ of two azomethine groups and of $\mathrm{S}$ of thioketo group. 
TABLe 1: Analytical, conductivity, molecular weight, and magnetic moment data of $\mathrm{Cu}^{2+}$ complexes of thiosemicarbazones.

\begin{tabular}{|c|c|c|c|c|c|c|c|c|c|}
\hline \multirow{2}{*}{ Complex } & \multirow{2}{*}{$\begin{array}{c}\text { Yield } \\
(\%)\end{array}$} & \multicolumn{4}{|c|}{ Analysis : Found (Calcd) \% } & \multirow{2}{*}{$\begin{array}{l}\text { mw Found } \\
\text { (Calcd) }\end{array}$} & \multirow{2}{*}{\multicolumn{2}{|c|}{$\begin{array}{ll} & \Lambda_{m} \\
\left(\mathrm{ohm}^{-1}\right. & \left.\mathrm{cm}^{2} \mathrm{~mole}^{-1}\right)\end{array}$}} & \multirow{2}{*}{$\begin{array}{l}\mu_{\text {eff }} \\
\mathrm{BM}\end{array}$} \\
\hline & & $\mathrm{Cu}$ & $\mathrm{N}$ & S & Anion & & & & \\
\hline $\mathrm{CuCl}_{2}\left(\mathrm{H}_{2} \mathrm{O}\right)$ BAAPTS & 75 & $\begin{array}{c}12.19 \\
(12.29)\end{array}$ & $\begin{array}{c}16.17 \\
(16.26)\end{array}$ & $\begin{array}{c}6.14 \\
(6.19)\end{array}$ & $\begin{array}{c}13.62 \\
(13.74)\end{array}$ & $\begin{array}{c}511 \\
(516.5)\end{array}$ & & 3.6 & 1.83 \\
\hline $\mathrm{CuBr}_{2}\left(\mathrm{H}_{2} \mathrm{O}\right)$ BAAPTS & 70 & $\begin{array}{c}10.38 \\
(10.48)\end{array}$ & $\begin{array}{c}13.73 \\
(13.87)\end{array}$ & $\begin{array}{c}5.23 \\
(5.28)\end{array}$ & $\begin{array}{c}26.22 \\
(26.42)\end{array}$ & $\begin{array}{c}599 \\
(605.5)\end{array}$ & & 3.7 & 1.92 \\
\hline $\mathrm{Cu}\left(\mathrm{NO}_{3}\right)_{2}\left(\mathrm{H}_{2} \mathrm{O}\right)$ BAAPTS & 75 & $\begin{array}{c}11.10 \\
(11.15)\end{array}$ & $\begin{array}{c}19.53 \\
(19.66)\end{array}$ & $\begin{array}{c}5.57 \\
(5.61)\end{array}$ & - & $\begin{array}{c}558 \\
(569.5)\end{array}$ & & 2.3 & 1.89 \\
\hline $\mathrm{Cu}(\mathrm{NCS})_{2}\left(\mathrm{H}_{2} \mathrm{O}\right) \mathrm{BAAPTS}$ & 70 & $\begin{array}{c}11.19 \\
(11.30)\end{array}$ & $\begin{array}{c}19.79 \\
(19.94)\end{array}$ & $\begin{array}{c}16.92 \\
(17.09)\end{array}$ & $\begin{array}{c}20.47 \\
(20.65)\end{array}$ & $\begin{array}{c}558 \\
(561.5)\end{array}$ & & 3.9 & 1.87 \\
\hline $\mathrm{Cu}(\mathrm{OAc})_{2}\left(\mathrm{H}_{2} \mathrm{O}\right) \mathrm{BAAPTS}$ & 67 & $\begin{array}{c}11.15 \\
(11.26)\end{array}$ & $\begin{array}{c}14.78 \\
(14.90)\end{array}$ & $\begin{array}{c}5.61 \\
(5.67)\end{array}$ & - & $\begin{array}{c}557 \\
(563.5)\end{array}$ & & 2.7 & 1.81 \\
\hline $\mathrm{CuCl}_{2}\left(\mathrm{H}_{2} \mathrm{O}\right)$ MBAAPTS & 73 & $\begin{array}{c}11.94 \\
(11.61)\end{array}$ & $\begin{array}{c}15.76 \\
(15.37)\end{array}$ & $\begin{array}{c}6.00 \\
(5.85)\end{array}$ & $\begin{array}{c}13.31 \\
(12.99)\end{array}$ & $\begin{array}{c}539 \\
(546.5)\end{array}$ & & 3.7 & 1.82 \\
\hline $\mathrm{CuBr}_{2}\left(\mathrm{H}_{2} \mathrm{O}\right) \mathrm{MBAAPTS}$ & 70 & $\begin{array}{l}10.19 \\
(9.99)\end{array}$ & $\begin{array}{c}13.49 \\
(13.21)\end{array}$ & $\begin{array}{c}5.13 \\
(5.03)\end{array}$ & $\begin{array}{c}25.74 \\
(25.17)\end{array}$ & $\begin{array}{c}629 \\
(635.5)\end{array}$ & & 3.9 & 1.92 \\
\hline $\mathrm{Cu}\left(\mathrm{NO}_{3}\right)_{2}\left(\mathrm{H}_{2} \mathrm{O}\right)$ MBAAPTS & 75 & $\begin{array}{c}10.83 \\
(10.39)\end{array}$ & $\begin{array}{c}19.13 \\
(18.68)\end{array}$ & $\begin{array}{c}5.44 \\
(5.33)\end{array}$ & - & $\begin{array}{c}590 \\
(599.5)\end{array}$ & & 5.6 & 1.82 \\
\hline $\mathrm{Cu}(\mathrm{NCS})_{2}\left(\mathrm{H}_{2} \mathrm{O}\right) \mathrm{MBAAPTS}$ & 70 & $\begin{array}{c}10.99 \\
(10.73)\end{array}$ & $\begin{array}{c}19.38 \\
(18.93)\end{array}$ & $\begin{array}{c}16.63 \\
(16.22)\end{array}$ & $\begin{array}{c}19.35 \\
(19.61)\end{array}$ & $\begin{array}{c}583 \\
(591.5)\end{array}$ & & 3.9 & 1.89 \\
\hline $\mathrm{Cu}(\mathrm{OAc})_{2}\left(\mathrm{H}_{2} \mathrm{O}\right) \mathrm{MBAAPTS}$ & 65 & $\begin{array}{c}10.95 \\
(10.69)\end{array}$ & $\begin{array}{c}14.48 \\
(14.15)\end{array}$ & $\begin{array}{c}5.51 \\
(5.39)\end{array}$ & - & $\begin{array}{c}587 \\
(593.5)\end{array}$ & & 2.7 & 1.84 \\
\hline $\mathrm{CuCl}_{2}\left(\mathrm{H}_{2} \mathrm{O}\right)$ DABAAPTS & 72 & $\begin{array}{c}11.26 \\
(11.34)\end{array}$ & $\begin{array}{c}17.39 \\
(17.51)\end{array}$ & $\begin{array}{c}5.65 \\
(5.71)\end{array}$ & $\begin{array}{c}12.52 \\
(12.68)\end{array}$ & $\begin{array}{c}552 \\
(559.5)\end{array}$ & & 3.3 & 1.82 \\
\hline $\mathrm{CuBr}_{2}\left(\mathrm{H}_{2} \mathrm{O}\right)$ DABAAPTS & 70 & $\begin{array}{c}9.69 \\
(9.79)\end{array}$ & $\begin{array}{c}15.01 \\
(15.11)\end{array}$ & $\begin{array}{c}4.87 \\
(4.93)\end{array}$ & $\begin{array}{c}24.47 \\
(24.67)\end{array}$ & $\begin{array}{c}644 \\
(648.5)\end{array}$ & & 3.7 & 1.92 \\
\hline $\mathrm{Cu}\left(\mathrm{NO}_{3}\right)_{2}\left(\mathrm{H}_{2} \mathrm{O}\right)$ DABAAPTS & 65 & $\begin{array}{c}10.29 \\
(10.36)\end{array}$ & $\begin{array}{c}20.67 \\
(20.89)\end{array}$ & $\begin{array}{c}5.16 \\
(5.22)\end{array}$ & - & $\begin{array}{c}607 \\
(612.5)\end{array}$ & & 3.0 & 1.81 \\
\hline $\mathrm{Cu}(\mathrm{NCS})_{2}\left(\mathrm{H}_{2} \mathrm{O}\right)$ DABAAPTS & 65 & $\begin{array}{c}10.42 \\
(10.50)\end{array}$ & $\begin{array}{c}20.69 \\
(20.84))\end{array}$ & $\begin{array}{c}15.75 \\
(15.88)\end{array}$ & $\begin{array}{c}19.03 \\
(19.18)\end{array}$ & $\begin{array}{c}598 \\
(604.5)\end{array}$ & & 5.3 & 1.87 \\
\hline $\mathrm{Cu}(\mathrm{OAc})_{2}\left(\mathrm{H}_{2} \mathrm{O}\right)$ DABAAPTS & 60 & $\begin{array}{c}10.37 \\
(10.46)\end{array}$ & $\begin{array}{c}16.07 \\
(16.15)\end{array}$ & $\begin{array}{c}5.18 \\
(5.27)\end{array}$ & - & $\begin{array}{c}602 \\
(606.5)\end{array}$ & & 4.7 & 1.83 \\
\hline $\mathrm{CuCl}_{2}\left(\mathrm{H}_{2} \mathrm{O}\right)$ CAAPTS & 73 & $\begin{array}{c}11.94 \\
(11.61)\end{array}$ & $\begin{array}{c}15.76 \\
(15.37)\end{array}$ & $\begin{array}{c}6.00 \\
(5.85)\end{array}$ & $\begin{array}{c}13.31 \\
(12.99)\end{array}$ & $\begin{array}{c}539 \\
(546.5)\end{array}$ & & 3.7 & 1.82 \\
\hline $\mathrm{CuBr}_{2}\left(\mathrm{H}_{2} \mathrm{O}\right)$ CAAPTS & 70 & $\begin{array}{l}10.19 \\
(9.99)\end{array}$ & $\begin{array}{c}13.49 \\
(13.21)\end{array}$ & $\begin{array}{c}5.13 \\
(5.03)\end{array}$ & $\begin{array}{l}25.74 \\
25.17\end{array}$ & $\begin{array}{c}539 \\
(546.5)\end{array}$ & & 3.6 & 1.92 \\
\hline $\mathrm{Cu}\left(\mathrm{NO}_{3}\right)_{2}\left(\mathrm{H}_{2} \mathrm{O}\right)$ CAAPTS & 75 & $\begin{array}{c}10.83 \\
(10.39)\end{array}$ & $\begin{array}{c}19.13 \\
(18.68)\end{array}$ & $\begin{array}{c}5.44 \\
(5.33)\end{array}$ & - & $\begin{array}{c}629 \\
(635.5)\end{array}$ & & 2.7 & 1.82 \\
\hline $\mathrm{Cu}(\mathrm{OAc})_{2}\left(\mathrm{H}_{2} \mathrm{O}\right) \mathrm{CAAPTS}$ & 65 & $\begin{array}{c}10.95 \\
(10.69)\end{array}$ & $\begin{array}{c}14.48 \\
(14.15)\end{array}$ & $\begin{array}{c}5.51 \\
(5.39)\end{array}$ & - & $\begin{array}{c}587 \\
(599.5)\end{array}$ & & 3.1 & 1.84 \\
\hline $\mathrm{Cu}(\mathrm{NCS})_{2}\left(\mathrm{H}_{2} \mathrm{O}\right) \mathrm{CAAPTS}$ & 70 & $\begin{array}{c}10.99 \\
(10.73)\end{array}$ & $\begin{array}{c}19.38 \\
(18.93)\end{array}$ & $\begin{array}{c}16.63 \\
(16.22)\end{array}$ & $\begin{array}{c}19.35 \\
(19.61)\end{array}$ & $\begin{array}{c}583 \\
(591.5)\end{array}$ & & 3.3 & 1.84 \\
\hline
\end{tabular}


TABLE 2: Antibacterial and antifungal activities of copper(II) complexes of BAAPTS and MBAAPTS.

\begin{tabular}{|c|c|c|c|c|c|c|}
\hline \multirow{3}{*}{ Complexes } & \multicolumn{4}{|c|}{ Antibacterial activity } & \multicolumn{2}{|c|}{ Antifungal activity } \\
\hline & \multicolumn{4}{|c|}{$(\text { Zone size in } \mathrm{mm})^{*}$} & \multirow[b]{2}{*}{ A niger } & \multirow[b]{2}{*}{ Calbicans } \\
\hline & Bs & $\mathrm{Sa}$ & $E c$ & St & & \\
\hline $\mathrm{CuCl}_{2}\left(\mathrm{H}_{2} \mathrm{O}\right)$ (BAAPTS $)$ & 12 & 11 & 11 & 12 & + & + \\
\hline $\mathrm{CuBr}_{2}\left(\mathrm{H}_{2} \mathrm{O}\right)(\mathrm{BAAPTS})$ & 11 & 10 & 11 & 09 & + & + \\
\hline $\mathrm{Cu}\left(\mathrm{NO}_{3}\right)_{2}\left(\mathrm{H}_{2} \mathrm{O}\right)(\mathrm{BAAPTS})$ & 13 & 10 & 10 & 10 & + & + \\
\hline $\mathrm{Cu}(\mathrm{NCS})_{2}\left(\mathrm{H}_{2} \mathrm{O}\right)(\mathrm{BAAPTS})$ & 13 & 12 & 11 & 10 & ++ & ++ \\
\hline $\mathrm{Cu}\left(\mathrm{CH}_{3} \mathrm{COO}\right)_{2}\left(\mathrm{H}_{2} \mathrm{O}\right)(\mathrm{BAAPTS})$ & 11 & 10 & 12 & 10 & + & + \\
\hline $\mathrm{CuCl}_{2}\left(\mathrm{H}_{2} \mathrm{O}\right)(\mathrm{MBAAPTS})$ & 15 & 12 & 13 & 14 & ++ & ++ \\
\hline $\mathrm{CuBr}_{2}\left(\mathrm{H}_{2} \mathrm{O}\right)$ (MBAAPTS) & 14 & 12 & 12 & 10 & ++ & ++ \\
\hline $\mathrm{Cu}\left(\mathrm{NO}_{3}\right)_{2}\left(\mathrm{H}_{2} \mathrm{O}\right)(\mathrm{MBAAPTS})$ & 15 & 11 & 11 & 10 & ++ & ++ \\
\hline $\mathrm{Cu}(\mathrm{NCS})_{2}\left(\mathrm{H}_{2} \mathrm{O}\right)(\mathrm{MBAAPTS})$ & 16 & 14 & 16 & 15 & ++ & + \\
\hline $\mathrm{Cu}\left(\mathrm{CH}_{3} \mathrm{COO}\right)_{2}\left(\mathrm{H}_{2} \mathrm{O}\right)(\mathrm{MBAAPTS})$ & 14 & 11 & 14 & 12 & ++ & ++ \\
\hline Ampicillin & 24 & 22 & 17 & 16 & - & - \\
\hline Tetracycline & 18 & 17 & 21 & 22 & - & - \\
\hline Salicylic acid & - & - & - & - & ++++ & ++++ \\
\hline
\end{tabular}

$*$ Result of representative experiments.

The strong bands observed at $3440-3270 \mathrm{~cm}^{-1}$ region in the free ligands have been assigned to $\nu(\mathrm{NH})$ vibrations. Practically no effect on these frequencies after complexation precludes the possibility of complexation at this group. The absorption at $\sim 1600 \mathrm{~cm}^{-1}$ in the free ligands can be attributed to $(\mathrm{C}=\mathrm{N})$ stretching vibrations of imine nitrogen, which is in agreement with the observations of previous authors [30, 31]. On complexation, these frequencies were observed to be shifted to lower wave number (Tables 3 and 4). These observations suggest involvement of unsaturated nitrogen atoms of the two azomethine groups in bonding with the metal ion. In substituted thioureas, the $(\mathrm{C}=\mathrm{S})$ stretching vibrations are contributed much with some other vibrations as $(\mathrm{CN})$ stretching and bending as well as $(\mathrm{N}-\mathrm{C}-\mathrm{S})$ bending modes [32]. In the spectra of the present ligands, the bands observed in $1330-1305 \mathrm{~cm}^{-1}$ region, $1120-1095$ $\mathrm{cm}^{-1}$, and $820-760 \mathrm{~cm}^{-1}$ regions are assigned to $[\nu(\mathrm{C}=\mathrm{S})$ $+\nu(\mathrm{C}=\mathrm{N})+\nu(\mathrm{C}-\mathrm{N})],[\delta(\mathrm{N}-\mathrm{C}-\mathrm{S})+\delta(\mathrm{C}=\mathrm{S})]$ bending and $\nu(\mathrm{C}=\mathrm{S}-)$ stretching, respectively, following the observations of Irving et al [33] and some other authors [34, 35]. Coordination of sulfur with metal ion would result in the displacement of elections toward the latter, thus resulting in the weakening of $(\mathrm{C}=\mathrm{S})$ bond. Hence, on complexation, $(\mathrm{C}=\mathrm{S})$ stretching vibrations should decrease and that of $(\mathrm{CN})$ should increase $[35,36]$. In all present complexes of $\mathrm{Cu}^{2+}$ with BAAPTS, MBAAPTS, DABAAPTS, and CAAPTS, the frequencies in $1330-1305 \mathrm{~cm}^{-1}$ get an increase by nearly
$30-60 \mathrm{~cm}^{-1}$. Similarly, bending modes of $(\mathrm{N}-\mathrm{C}-\mathrm{S})$ and $(\mathrm{C}=\mathrm{S})$ also get increase, but in lesser amount. On the other hand, on complexation, the frequencies in $820-760 \mathrm{~cm}^{-1}$ are shifted to lower wave numbers and intensity of the bands are also reduced. All these peculiar changes on complexation confidently precludes any unambigious ascertain of metalsulfur bond.

The possibility of thione-thiol tautomerism $(\mathrm{H}-\mathrm{N}-$ $\mathrm{C}=\mathrm{S})(\mathrm{C}=\mathrm{N}-\mathrm{SH})$ in these ligands has been ruled out for no bands around $2700-2500 \mathrm{~cm}^{-1}$, characteristic of thiol group is displayed in the infrared absorption $[37,38]$. In far infrared region, the bands in $410-330 \mathrm{~cm}^{-1}$ are tentatively assigned to $\nu(\mathrm{Cu}-\mathrm{N}) / v(\mathrm{Cu}-\mathrm{S})$ (metal-ligand) stretching bands [39-41]. In conclusion, the infrared spectral studies suggest the tridentate $(\mathrm{N}, \mathrm{N}, \mathrm{S})$ nature by pointing out the sites of possible donor atoms.

The presence of coordinated water was suggested by the very broad absorption centered around $3450 \mathrm{~cm}^{-1}$ in the infrared spectra. Bands at $\sim 930$ and $770 \mathrm{~cm}^{-1}$ may be attributed to rocking and wagging modes of the coordinated water [42].

\section{ANIONS}

The pseudohalide $\left(\mathrm{SCN}^{-}\right)$ion is a very interesting anion since it may coordinate through the sulfur (thio-) or through the nitrogen (isothio-) or through both these atoms 
TABLE 3: Key IR bands $\left(\mathrm{cm}^{-1}\right)$ of BAAPTS and MBAAPTS and their $\mathrm{Cu}^{2+}$ complexes.

\begin{tabular}{|c|c|c|c|c|c|c|c|}
\hline \multirow[b]{2}{*}{ Compounds } & \multicolumn{7}{|c|}{ Assignments } \\
\hline & $v(\mathrm{NH})$ & $v(\mathrm{C}=\mathrm{N})$ & $\begin{array}{c}\nu(\mathrm{C}=\mathrm{S})+\nu(\mathrm{C}+\mathrm{N}) \\
\quad+v(\mathrm{C}-\mathrm{N})\end{array}$ & $\begin{array}{c}\delta(\mathrm{NCS})+\mathrm{CS}- \\
\text { bending }\end{array}$ & $v(\mathrm{~N}-\mathrm{N})$ & $\nu(\mathrm{C}=\mathrm{S})$ & $\begin{array}{l}\nu(\mathrm{Cu}-\mathrm{N}) / \\
v(\mathrm{Cu}-\mathrm{S})\end{array}$ \\
\hline \multirow{2}{*}{ BAAPTS } & $3440 \mathrm{~s}$ & 1600 vs & $1330 \mathrm{~s}$ & $1120 \mathrm{~m}$ & $1050 \mathrm{~m}$ & $820 \mathrm{~s}$ & - \\
\hline & $3270 \mathrm{~m}$ & - & $1305 \mathrm{~s}$ & $1095 \mathrm{~m}$ & - & $760 \mathrm{vs}$ & - \\
\hline \multirow{2}{*}{$\mathrm{CuCl}_{2}(\mathrm{BAAPTS}) \mathrm{H}_{2} \mathrm{O}$} & $3442 \mathrm{~s}$ & $1575 \mathrm{~s}$ & $1390 \mathrm{~m}$ & $1150 \mathrm{~m}$ & $1060 \mathrm{~m}$ & $780 \mathrm{~s}$ & $420 \mathrm{~m}$ \\
\hline & $3275 \mathrm{~m}$ & - & $1345 \mathrm{~m}$ & $1130 \mathrm{~m}$ & - & $740 \mathrm{~m}$ & $310 \mathrm{~m}$ \\
\hline \multirow{2}{*}{$\mathrm{CuBr}_{2}$ (BAAPTS) $\mathrm{H}_{2} \mathrm{O}$} & $3445 \mathrm{~m}$ & $1572 \mathrm{~s}$ & $1365 \mathrm{~m}$ & $1160 \mathrm{~m}$ & $1065 \mathrm{~m}$ & $770 \mathrm{~s}$ & $405 \mathrm{~m}$ \\
\hline & $3280 \mathrm{~m}$ & - & $1335 \mathrm{~m}$ & $1135 \mathrm{~m}$ & - & $745 \mathrm{~m}$ & $318 w$ \\
\hline \multirow{2}{*}{$\mathrm{Cu}\left(\mathrm{NO}_{3}\right)_{2}(\mathrm{BAAPTS}) \mathrm{H}_{2} \mathrm{O}$} & $3400 s$ & $1560 s$ & $1375 \mathrm{~m}$ & $1160 \mathrm{~m}$ & $1062 \mathrm{~m}$ & $755 \mathrm{~s}$ & $415 \mathrm{~m}$ \\
\hline & $3272 \mathrm{~m}$ & - & $1340 \mathrm{~m}$ & $1135 \mathrm{~m}$ & - & $730 \mathrm{~s}$ & $310 \mathrm{w}$ \\
\hline \multirow{2}{*}{$\mathrm{Cu}(\mathrm{NCS})_{2}(\mathrm{BAAPTS}) \mathrm{H}_{2} \mathrm{O}$} & $3445 \mathrm{~s}$ & $1565 \mathrm{~s}$ & $1370 \mathrm{~m}$ & $1165 \mathrm{~m}$ & $1065 \mathrm{~m}$ & $772 \mathrm{~s}$ & $410 \mathrm{~m}$ \\
\hline & $3270 \mathrm{~m}$ & - & $1345 \mathrm{~m}$ & $1140 \mathrm{~m}$ & - & $745 \mathrm{~m}$ & $305 w$ \\
\hline \multirow{2}{*}{$\mathrm{Cu}(\mathrm{OAc})_{2}(\mathrm{BAAPTS}) \mathrm{H}_{2} \mathrm{O}$} & $3445 \mathrm{~s}$ & $1560 \mathrm{~s}$ & $1370 \mathrm{~m}$ & $1155 \mathrm{~m}$ & $1060 \mathrm{~m}$ & $770 \mathrm{~m}$ & $412 \mathrm{~m}$ \\
\hline & $3275 \mathrm{~m}$ & - & $1340 \mathrm{~m}$ & $1130 \mathrm{~m}$ & - & $740 \mathrm{~m}$ & $305 \mathrm{w}$ \\
\hline \multirow{2}{*}{ MBAAPTS } & $3420 \mathrm{~s}$ & 1600 vs & $1320 s$ & $1120 \mathrm{~m}$ & $1060 \mathrm{~m}$ & $840 \mathrm{~s}$ & 一 \\
\hline & $3310 s$ & - & $1195 \mathrm{~m}$ & $1095 \mathrm{~m}$ & - & $820 \mathrm{~s}$ & 一 \\
\hline \multirow{2}{*}{$\mathrm{CuCl}_{2}(\mathrm{MBAAPTS}) \mathrm{H}_{2} \mathrm{O}$} & $3415 \mathrm{~m}$ & $1575 \mathrm{~s}$ & $1365 \mathrm{~s}$ & $1170 \mathrm{~m}$ & $1072 \mathrm{~m}$ & $772 \mathrm{~s}$ & $415 \mathrm{~m}$ \\
\hline & $3312 \mathrm{~m}$ & - & $1240 \mathrm{~m}$ & $1130 \mathrm{~m}$ & - & $755 \mathrm{~s}$ & $302 \mathrm{w}$ \\
\hline \multirow{2}{*}{$\mathrm{CuBr}_{2}\left(\mathrm{MBAAPTS} \mathrm{H}_{2} \mathrm{O}\right.$} & $3422 \mathrm{~m}$ & $1570 s$ & $1360 s$ & $1172 \mathrm{~m}$ & $1070 \mathrm{~m}$ & $770 \mathrm{~s}$ & $398 \mathrm{~m}$ \\
\hline & $3310 \mathrm{~m}$ & - & $1245 \mathrm{~m}$ & $1125 \mathrm{~m}$ & - & $750 \mathrm{~s}$ & $300 \mathrm{w}$ \\
\hline \multirow{2}{*}{$\mathrm{Cu}\left(\mathrm{NO}_{3}\right)_{2}(\mathrm{MBAAPTS}) \mathrm{H}_{2} \mathrm{O}$} & $3418 \mathrm{~m}$ & $1562 \mathrm{~s}$ & $1362 \mathrm{~s}$ & $1175 \mathrm{~m}$ & $1068 \mathrm{~m}$ & $771 \mathrm{~s}$ & $408 \mathrm{~m}$ \\
\hline & $3310 \mathrm{~m}$ & - & $1242 \mathrm{~m}$ & $1130 \mathrm{~m}$ & - & $752 \mathrm{~s}$ & $305 \mathrm{w}$ \\
\hline \multirow{2}{*}{$\mathrm{Cu}(\mathrm{NCS})_{2}(\mathrm{MBAAPTS}) \mathrm{H}_{2} \mathrm{O}$} & $3415 \mathrm{~m}$ & $1565 \mathrm{~s}$ & $1362 \mathrm{~s}$ & $1172 \mathrm{~m}$ & $1072 \mathrm{~m}$ & $770 \mathrm{~s}$ & $415 \mathrm{~m}$ \\
\hline & $3315 w$ & - & $1240 \mathrm{~m}$ & $1130 \mathrm{~m}$ & - & $745 \mathrm{~s}$ & $310 w$ \\
\hline \multirow{2}{*}{$\mathrm{Cu}(\mathrm{OAc})_{2}(\mathrm{MBAAPTS})$} & $3415 \mathrm{~m}$ & $1568 s$ & $1370 \mathrm{~s}$ & $1172 \mathrm{~m}$ & $1070 \mathrm{~m}$ & $782 \mathrm{~s}$ & $410 \mathrm{~m}$ \\
\hline & $3315 \mathrm{~m}$ & - & $1245 \mathrm{~m}$ & $1125 \mathrm{~m}$ & - & $755 \mathrm{~m}$ & $312 \mathrm{w}$ \\
\hline
\end{tabular}

(bridging). The various criteria proposed for determining the mode of bonding have been discussed by Nakamoto [42]. In general, the bonding depends on (a) the nature of the central atom, (b) the nature of other ligands in the coordination sphere, and (c) environmental controls and kinetic (mechanistic) controls. In the present complexes, the frequencies in $2045-2035 \mathrm{~cm}^{-1}$ due to $(\mathrm{C}-\mathrm{N})$ stretch $\left(\nu_{1}\right), 845-835 \mathrm{~cm}^{-1}$ due to $\nu(\mathrm{C}-\mathrm{S})$ stretch $\left(\nu_{2}\right)$, and $465-$ $400 \mathrm{~cm}^{-1}$ for $\delta$ (NCS) have been identified. These frequencies are associated with the terminal N-bonded isothiocyanate ions $[43,44]$. In nitrate complexes, the occurrence of two strong bands in $1550-1535 \mathrm{~cm}^{-1}$ and $1315-1300 \mathrm{~cm}^{-1}$ are attributed to $v_{4}$ and $v_{1}$ modes of vibrations of covalently bonded nitrate groups, respectively. This suggests that nitrate groups are present inside the coordination sphere $[45,46]$. Other absorptions associated with covalently bonded nitrate groups are also observed in the spectra of these complexes. If the $\left(v_{4}-v_{1}\right)$ difference is taken as an approximate measure of the covalency of nitrate group $[46,47]$, a value of $\sim 200 \mathrm{~cm}^{-1}$ for the complexes studied suggests strong covalency for the metal-nitrate bonding. Lever et al $[48,49]$ have shown that the number and relative energies of nitrate combination frequencies $\left(\nu_{1}+\nu_{4}\right)$ in the $1800-1700 \mathrm{~cm}^{-1}$ region of the infrared spectrum, may be used as an aid to distinguish the various coordination's modes of the nitrate group. According to Lever et al $[48,49]$, bidentate coordination involves a greater 
TABLE 4: Key IR bands $\left(\mathrm{cm}^{-1}\right)$ of DABAAPTS and CAAPTS and their $\mathrm{Cu}^{2+}$ complexes.

\begin{tabular}{|c|c|c|c|c|c|c|c|}
\hline Compounds & $\nu(\mathrm{NH})$ & $\nu(\mathrm{C}=\mathrm{N})$ & $\begin{array}{c}\nu(\mathrm{C}=\mathrm{S})+\nu(\mathrm{C}=\mathrm{N}) \\
+v(\mathrm{C}-\mathrm{N})\end{array}$ & $\begin{array}{c}\delta(\mathrm{NCS})+\mathrm{CS} \\
\text { bending }\end{array}$ & $\nu(\mathrm{N}-\mathrm{N})$ & $\nu(\mathrm{C}=\mathrm{S})$ & $\begin{array}{l}\nu(\mathrm{Cu}-\mathrm{N}) / \\
\nu(\mathrm{Cu}-\mathrm{S})\end{array}$ \\
\hline \multirow{2}{*}{ DABAAPTS } & $3360 \mathrm{~s}$ & $1600 \mathrm{vs}$ & $1310 s$ & 1115 & $1050 \mathrm{~m}$ & $830 \mathrm{~s}$ & - \\
\hline & $3330 \mathrm{~m}$ & - & $1290 \mathrm{~s}$ & 1095 & - & $730 \mathrm{~s}$ & - \\
\hline \multirow{2}{*}{$\mathrm{CuCl}_{2}($ DABAAPTS $) \mathrm{H}_{2} \mathrm{O}$} & $3362 \mathrm{~s}$ & 1572 vs & $1365 \mathrm{~m}$ & 1165 & $1065 \mathrm{~m}$ & $782 \mathrm{~m}$ & $410 \mathrm{~m}$ \\
\hline & $3320 \mathrm{~m}$ & - & $1340 \mathrm{~m}$ & 1132 & - & $710 \mathrm{~m}$ & $305 \mathrm{w}$ \\
\hline \multirow{2}{*}{$\mathrm{CuBr}_{2}(\mathrm{DABAAPTS}) \mathrm{H}_{2} \mathrm{O}$} & $3360 s$ & $1570 \mathrm{~s}$ & $1370 \mathrm{~m}$ & 1172 & $1068 \mathrm{~m}$ & $775 \mathrm{~m}$ & $415 \mathrm{~m}$ \\
\hline & $3335 \mathrm{~m}$ & - & $1330 \mathrm{~m}$ & 1130 & - & $710 \mathrm{~m}$ & $300 w$ \\
\hline \multirow{2}{*}{$\mathrm{Cu}\left(\mathrm{NO}_{3}\right)_{2}(\mathrm{DABAAPTS}) \mathrm{H}_{2} \mathrm{O}$} & $3360 \mathrm{~s}$ & $1575 s$ & $1365 \mathrm{~m}$ & 1160 & $1062 \mathrm{~m}$ & $770 \mathrm{~m}$ & $415 \mathrm{~m}$ \\
\hline & $3332 \mathrm{~m}$ & - & $1342 \mathrm{~m}$ & 1142 & - & $705 \mathrm{~m}$ & $302 \mathrm{w}$ \\
\hline \multirow{2}{*}{$\mathrm{Cu}(\mathrm{NCS})_{2}(\mathrm{DABAAPTS}) \mathrm{H}_{2} \mathrm{O}$} & $3362 \mathrm{~s}$ & 1565 vs & $1372 \mathrm{~s}$ & 1165 & $1060 \mathrm{~m}$ & $775 \mathrm{~m}$ & $400 \mathrm{~m}$ \\
\hline & $3330 \mathrm{~m}$ & - & $1345 \mathrm{~m}$ & 1130 & - & $722 \mathrm{~m}$ & $305 \mathrm{w}$ \\
\hline \multirow{2}{*}{$\mathrm{Cu}(\mathrm{OAc})_{2}(\mathrm{DABAAPTS}) \mathrm{H}_{2} \mathrm{O}$} & $3365 s$ & $1565 \mathrm{~s}$ & $1370 s$ & 1162 & $1065 \mathrm{~m}$ & $770 \mathrm{~m}$ & $410 \mathrm{~m}$ \\
\hline & $3335 \mathrm{~m}$ & - & $1340 \mathrm{~m}$ & 1135 & - & $715 \mathrm{~m}$ & $310 \mathrm{w}$ \\
\hline \multirow{2}{*}{ CAAPTS } & $3315 s$ & 1605 vs & $1320 \mathrm{~s}$ & 1125 & $1060 \mathrm{~m}$ & $840 \mathrm{~m}$ & - \\
\hline & $3200 \mathrm{~m}$ & - & $1295 \mathrm{~s}$ & 1090 & - & $770 \mathrm{~m}$ & - \\
\hline \multirow{2}{*}{$\mathrm{CuCl}_{2}(\mathrm{CAAPTS}) \mathrm{H}_{2} \mathrm{O}$} & $3320 \mathrm{~s}$ & $1555 \mathrm{~s}$ & $1370 \mathrm{~m}$ & 1180 & $1070 \mathrm{~m}$ & $790 \mathrm{~m}$ & $415 \mathrm{~m}$ \\
\hline & $3205 \mathrm{~m}$ & - & $1330 \mathrm{~m}$ & 1140 & - & $755 \mathrm{~m}$ & $302 \mathrm{w}$ \\
\hline \multirow{2}{*}{$\mathrm{CuBr}_{2}(\mathrm{CAAPTS}) \mathrm{H}_{2} \mathrm{O}$} & $3315 s$ & 1565 vs & $1365 s$ & 1175 & $1068 \mathrm{~m}$ & $795 \mathrm{~m}$ & $410 \mathrm{~m}$ \\
\hline & $3202 \mathrm{~m}$ & - & $1325 \mathrm{~m}$ & 1135 & - & $750 \mathrm{~m}$ & $335 \mathrm{w}$ \\
\hline \multirow{2}{*}{$\mathrm{Cu}\left(\mathrm{NO}_{3}\right)_{2}(\mathrm{CAAPTS}) \mathrm{H}_{2} \mathrm{O}$} & $3315 s$ & $1572 \mathrm{~s}$ & $1375 \mathrm{~s}$ & 1170 & $1072 \mathrm{~m}$ & $792 \mathrm{~m}$ & $402 \mathrm{~m}$ \\
\hline & $3205 \mathrm{~m}$ & - & $1335 \mathrm{~m}$ & 1130 & - & $745 \mathrm{~m}$ & $305 \mathrm{w}$ \\
\hline \multirow{2}{*}{$\mathrm{Cu}(\mathrm{NCS})_{2}(\mathrm{CAAPTS}) \mathrm{H}_{2} \mathrm{O}$} & $3312 \mathrm{~s}$ & $1570 s$ & $1360 \mathrm{~m}$ & 1172 & $1075 \mathrm{~m}$ & $780 \mathrm{~m}$ & $415 \mathrm{~m}$ \\
\hline & $3200 \mathrm{~m}$ & - & $1330 \mathrm{~m}$ & 1125 & - & $748 \mathrm{~m}$ & $300 \mathrm{w}$ \\
\hline \multirow{2}{*}{$\mathrm{Cu}(\mathrm{OAc})_{2}(\mathrm{CAAPTS}) \mathrm{H}_{2} \mathrm{O}$} & $3315 s$ & $1570 \mathrm{~s}$ & $1355 \mathrm{~m}$ & 1170 & $1070 \mathrm{~m}$ & $795 \mathrm{~m}$ & $408 \mathrm{~m}$ \\
\hline & $3205 \mathrm{~m}$ & - & $1320 \mathrm{~m}$ & 1130 & - & $750 \mathrm{~m}$ & $303 \mathrm{w}$ \\
\hline
\end{tabular}

distortion from $\mathrm{D}_{3 \mathrm{~h}}$ symmetry than unidentate coordination, therefore, bidentate complexes should show a larger separation of $\left(\nu_{1}+v_{4}\right)$. The authors have tried to apply this method to the present complexes. In all present complexes, a separation of $15-25 \mathrm{~cm}^{-1}$ in the combination bands $\left(\nu_{1}+\right.$ $\left.v_{4}\right)$ in the $1800-1700 \mathrm{~cm}^{-1}$ region concludes the monodentate nitrate coordination.

\section{ELECTRONIC SPECTRA}

Electronic spectra data of all copper(II) complexes are collected in Table 5. The spectra of these complexes consist of a broad band $\left(16000 \pm 200 \mathrm{~cm}^{-1}\right)$ of medium intensity in the visible region which can be identified as a $d-d$ band of the central ion, that is, an electronic transition mainly localized on $\mathrm{Cu}(\mathrm{II})$. The spectra of tetragonality distorted complexes should consist of three bands corresponding to the transitions ${ }^{2} \mathrm{~B}_{1 \mathrm{~g}} \rightarrow{ }^{2} \mathrm{~A}_{2},{ }^{2} \mathrm{~B}_{1 \mathrm{~g}} \rightarrow{ }^{2} \mathrm{~B}_{2 \mathrm{~g}}$, and ${ }^{2} \mathrm{~B}_{1 \mathrm{~g}} \rightarrow 2 \mathrm{E}_{\mathrm{g}}$ in order to increase energy. But generally, such complexes exhibit $[50,51]$, a broad structureless band with or without shoulder between $14000-18000 \mathrm{~cm}^{-1}$ depending upon the strength of in-plane and axial ligands. Since only a single $d-d$ broad band at $16000 \pm 200 \mathrm{~cm}^{-1}$ has been observed in the complexes reported herein, it is concluded that all three transitions be within this broad envelope. The calculated $10 \mathrm{Dq}$ values are also included in Table 5.

\section{THERMAL STUDIES}

Comparatively less is known about the thermal properties of transition metal complexes of thiosemicarbazones [39-41, 52-54]. In the present work, we report herein 
TABLE 5: Electronic spectral bands $\left(\mathrm{cm}^{-1}\right)$ of $\mathrm{Cu}^{2+}$ complexes of thiosemicarbazones.

\begin{tabular}{|c|c|c|c|c|}
\hline \multirow{2}{*}{$\begin{array}{l}\text { Complex } \\
\mathrm{CuCl}_{2}(\mathrm{BAAPTS}) \mathrm{H}_{2} \mathrm{O}\end{array}$} & \multirow{2}{*}{$\frac{(d-d) \text { band }}{15800}$} & \multicolumn{2}{|c|}{ CT-bands } & \multirow{2}{*}{$\frac{10 \mathrm{Dq}}{7900}$} \\
\hline & & 23000 & 28700 & \\
\hline $\mathrm{CuBr}_{2}(\mathrm{BAAPTS}) \mathrm{H}_{2} \mathrm{O}$ & 16250 & 23500 & 28600 & 8125 \\
\hline $\mathrm{Cu}\left(\mathrm{NO}_{3}\right)_{2}(\mathrm{BAAPTS}) \mathrm{H}_{2} \mathrm{O}$ & 16100 & 22900 & 28700 & 8050 \\
\hline $\mathrm{Cu}(\mathrm{NCS})_{2}(\mathrm{BAAPTS}) \mathrm{H}_{2} \mathrm{O}$ & 16200 & 23200 & 28700 & 8100 \\
\hline $\mathrm{Cu}(\mathrm{OAc})_{2}(\mathrm{BAAPTS}) \mathrm{H}_{2} \mathrm{O}$ & 16300 & 23000 & 28800 & 8150 \\
\hline $\mathrm{CuCl}_{2}(\mathrm{MBAAPTS}) \mathrm{H}_{2} \mathrm{O}$ & 16000 & 23000 & 26600 & 8000 \\
\hline $\mathrm{CuBr}_{2}(\mathrm{MBAAPTS}) \mathrm{H}_{2} \mathrm{O}$ & 16000 & 23000 & 26900 & 8000 \\
\hline $\mathrm{Cu}\left(\mathrm{NO}_{3}\right)_{2}(\mathrm{MBAAPTS}) \mathrm{H}_{2} \mathrm{O}$ & 16300 & 23000 & 28700 & 8150 \\
\hline $\mathrm{Cu}(\mathrm{NCS})_{2}(\mathrm{MBAAPTS}) \mathrm{H}_{2} \mathrm{O}$ & 16000 & 23000 & 28850 & 8000 \\
\hline $\mathrm{Cu}(\mathrm{OAc})_{2}(\mathrm{MBAAPTS}) \mathrm{H}_{2} \mathrm{O}$ & 16100 & 22900 & 28750 & 8050 \\
\hline $\mathrm{CuCl}_{2}(\mathrm{DABAAPTS}) \mathrm{H}_{2} \mathrm{O}$ & 15800 & 23100 & 28770 & 7900 \\
\hline $\mathrm{CuBr}_{2}(\mathrm{DABAAPTS}) \mathrm{H}_{2} \mathrm{O}$ & 16060 & 23080 & 28700 & 8030 \\
\hline $\mathrm{Cu}\left(\mathrm{NO}_{3}\right)_{2}(\mathrm{DABAAPTS}) \mathrm{H}_{2} \mathrm{O}$ & 16000 & 23000 & 28750 & 8000 \\
\hline $\mathrm{Cu}(\mathrm{NCS})_{2}(\mathrm{DABAAPTS}) \mathrm{H}_{2} \mathrm{O}$ & 16150 & 23010 & 26900 & 8075 \\
\hline $\mathrm{Cu}(\mathrm{OAc})_{2}(\mathrm{DABAAAPTS}) \mathrm{H}_{2} \mathrm{O}$ & 15800 & 23080 & 28770 & 7900 \\
\hline $\mathrm{CuCl}_{2}(\mathrm{CAAPTS}) \mathrm{H}_{2} \mathrm{O}$ & 16300 & 23200 & 28700 & 8150 \\
\hline $\mathrm{CuBr}_{2}(\mathrm{CAAPTS}) \mathrm{H}_{2} \mathrm{O}$ & 16200 & 23200 & 28700 & 8100 \\
\hline $\mathrm{Cu}\left(\mathrm{NO}_{3}\right)_{2}(\mathrm{CAAPTS}) \mathrm{H}_{2} \mathrm{O}$ & 15800 & 22900 & 28700 & 7900 \\
\hline $\mathrm{Cu}(\mathrm{NCS})_{2}(\mathrm{CAAPTS}) \mathrm{H}_{2} \mathrm{O}$ & 16150 & 23000 & 26600 & 8075 \\
\hline $\mathrm{Cu}(\mathrm{OAc})_{2}(\mathrm{CAAPTS}) \mathrm{H}_{2} \mathrm{O}$ & 16200 & 23100 & 28700 & 8150 \\
\hline
\end{tabular}

TABLE 6: Thermal decomposition data for $\mathrm{Cu}^{2+}$ complexes of BAAPTS and CAAPTS.

\begin{tabular}{|c|c|c|c|c|c|}
\hline Complex & $\begin{array}{c}\text { Stage } \\
\text { of } \\
\text { decomposition }\end{array}$ & Reaction & $\begin{array}{c}\text { Peak } \\
\text { temp }\left({ }^{\circ} \mathrm{C}\right) \text { in } \\
\text { dtg }\end{array}$ & $\begin{array}{c}\text { Temp } \\
\text { range in } \\
\operatorname{dtg}\left({ }^{\circ} \mathrm{C}\right)\end{array}$ & $\begin{array}{c}\text { Peak } \\
\text { temp }\left({ }^{\circ} \mathrm{C}\right) \\
\text { in dta }\end{array}$ \\
\hline \multirow{4}{*}{$\mathrm{Cu}(\mathrm{BAAPTS}) \mathrm{H}_{2} \mathrm{O}\left(\mathrm{NO}_{3}\right)_{2}$} & I & $\mathrm{Cu}(\mathrm{BAAPTS}) \mathrm{H}_{2} \mathrm{O}\left(\mathrm{NO}_{3}\right)_{2} \rightarrow \mathrm{Cu}(\mathrm{BAAPTS})\left(\mathrm{NO}_{3}\right)_{2}$ & 140 & $105-170$ & 150 (endo) \\
\hline & II & $\mathrm{Cu}(\mathrm{BAAPTS})\left(\mathrm{NO}_{3}\right)_{2} \rightarrow \mathrm{Cu}(\mathrm{BAAPTS})_{0.5}\left(\mathrm{NO}_{3}\right)_{2}$ & 340 & $295-380$ & 335 (exo) \\
\hline & III & $\mathrm{Cu}(\mathrm{BAAPTS})_{0.5}\left(\mathrm{NO}_{3}\right)_{2} \rightarrow \mathrm{Cu}\left(\mathrm{NO}_{3}\right)_{2}$ & 540 & $500-590$ & 525 (exo) \\
\hline & IV & $\mathrm{Cu}\left(\mathrm{NO}_{3}\right)_{2} \rightarrow \mathrm{CuO}$ & 730 & $685-770$ & $740($ exo $)$ \\
\hline \multirow{4}{*}{$\mathrm{Cu}(\mathrm{CAAPTS}) \mathrm{H}_{2} \mathrm{O}(\mathrm{OAc})_{2}$} & I & $\left.\mathrm{Cu}(\mathrm{CAAPTS}) \mathrm{H}_{2} \mathrm{O}(\mathrm{OAc})_{2} \rightarrow \mathrm{Cu}(\mathrm{CAAPTS}) \mathrm{OAc}\right)_{2}$ & 150 & $120-160$ & 135 (endo) \\
\hline & II & $\mathrm{Cu}(\mathrm{CAAPTS})(\mathrm{OAc})_{2} \rightarrow \mathrm{Cu}(\mathrm{CAAPTS})_{0.5}(\mathrm{OAc})_{2}$ & 350 & $310-395$ & 360 (exo) \\
\hline & III & $\mathrm{Cu}(\mathrm{CAAPTS})_{0.5}(\mathrm{OAc})_{2} \rightarrow \mathrm{Cu}(\mathrm{OAc})_{2}$ & 550 & $510-600$ & 535 (exo) \\
\hline & IV & $\mathrm{Cu}(\mathrm{OAc})_{2} \rightarrow \mathrm{CuO}$ & 710 & $670-755$ & 720 (exo) \\
\hline
\end{tabular}

the thermal decomposition data of the two representative complexes, that is, $\left[\mathrm{Cu}(\mathrm{BAAPTS}) \mathrm{H}_{2} \mathrm{O}\left(\mathrm{NO}_{3}\right)_{2}\right]$ and $\left[\mathrm{Cu}(\mathrm{CAAPTS}) \mathrm{H}_{2} \mathrm{O}\left(\mathrm{CH}_{3} \mathrm{COO}\right)_{2}\right]$, presented in Table 6. The careful analyses of thermogravimetric curves suggest that both complexes contain one molecule of coordinated water, which is evident by loss in weight at $\sim 160^{\circ} \mathrm{C}$. There is no change upto $\sim 300^{\circ} \mathrm{C}$ after that there is a break in the curves due to evaporation of 0.5 molecule of organic ligand, the remaining ligand is removed from the coordination sphere at $\sim 600^{\circ} \mathrm{C}$. Finally, at $\sim 760^{\circ} \mathrm{C}, \mathrm{CuO}$ is formed $[37,38]$. 


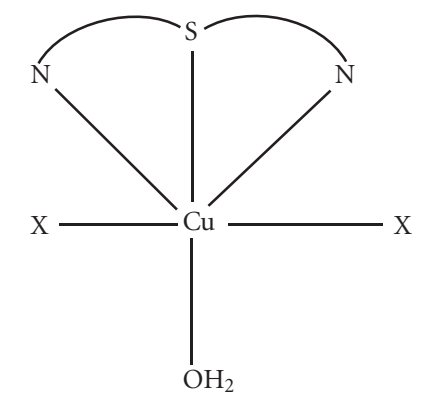

$\left(\mathrm{X}=\mathrm{CL}, \mathrm{Br}, \mathrm{NO}_{3}, \mathrm{NCS}\right.$, or $\left.\mathrm{OOCCH}_{3}\right)$

Scheme 1: $\left(\mathrm{X}=\mathrm{Cl}, \mathrm{Br}, \mathrm{NO}_{3}, \mathrm{NCS}\right.$, or $\left.\mathrm{OOCCH}_{3}\right)$.

The probable structures of the present complexes are suggested as in Scheme 1.

\section{CONCLUSION}

Thus in the present studies, all the thiosemicarbazones are coordinating to $\mathrm{Cu}^{2+}$ ion as neutral tridentate $(\mathrm{N}, \mathrm{N}, \mathrm{S})$ ligands. The magnetic and elecytronic spectral studies suggest the distorted octahedral geometries of the present complexes. Copper(II) complexes of BAAPTS and MBAAPTS have moderate antibacterial activity against $E$ coli and $S$ typhi and antifungal activity against $A$ niger and $C$ albicans.

\section{REFERENCES}

[1] Padhyé SB, Kauffman GB. Transition metal complexes of semicarbazones and thiosemicarbazones. Coordination Chemistry Reviews. 1985;63:127-160.

[2] West DX, Padhyé SB, Sonawane PB, Chikte RC. Structural and physical correlation in the biological properties of transition metal N-heterocyclic thiosemicarbazones and Salkyldithiocarbazate complexes. Structure \& Bonding. 1991; 76:1.

[3] West DX, Padhyé SB, Sonawane PB, Chikate RC. Copper(II) complexes of tridentate $(\mathrm{O}, \mathrm{N}, \mathrm{S})$ thiosemicarbazones. Asian Journal of Chemistry Reviews. 1990;4(1):125.

[4] Nandi AK, Chaudhri S, Mazumdah SK, Ghosh S. Effect of chlorine substitution on the structure and activity of 4phenylthiosemicarbazide: crystal and molecular structure of 4-(4-chlorophenyl)thiosemicarbazide. Journal of the Chemical Society, Perkin Transactions 2. 1984;(11):1729-1733.

[5] Ali MA, Chowdhary DA, Naziruddin M. Four- and five-coordinate copper(II) complexes containing mixed ligands. Polyhedron. 1984;3(5):595-598.

[6] Scovill JP, Klayman DL, Franchino CF. 2-acetylpyridine thiosemicarbazones. 4. Complexes with transition metals as antimalarial and antileukemic agents. Journal of Medicinal Chemistry. 1982;25(10):1261-1264.

[7] Hossain ME, Alam MN, Begum J, et al. The preparation, characterization, crystal structure and biological activities of some copper(II) complexes of the 2-benzoylpyridine Schiff bases of S-methyl- and S-benzyldithiocarbazate. Inorganica Chimica Acta. 1996;249(2):207-213.
[8] Bindu P, Kurup MRP, Satyakeerty TR. Epr, cyclic voltammetric and biological activities of copper(II) complexes of salicylaldehyde N(4)-substituted thiosemicarbazone and heterocyclic bases. Polyhedron. 1999;18(3-4):321-331.

[9] Agarwal RK, Singh G, Bhushan B. Synthesis and characterization of palladium(II) complexes of $4[\mathrm{~N}$-(cinnamalidene)amino] antipyrine thiosemicarbazone. Polish Journal of Chemistry. 1994;68:871-874.

[10] Agarwal RK, Prasad S, Gahlot N. Synthesis, spectral and thermal properties of some penta-coordinated complexes of oxovanadium(IV) derived from thiosemicarbazones of 4-aminoantipyrine. Turkish Journal of Chemistry. 2004;28(6): 691-702.

[11] Agarwal RK, Singh G, Bhushan B. Synthesis and biocidal activity of some new thiosemicarbazones of 4-aminoantipyrine. Journal of the Institution of Chemists (India). 1993;65:133-135.

[12] Cordes EH, Jencks WP. On the mechanism of Schiff base formation and hydrolysis. Journal of the American Chemical Society. 1962;84(5):832-837.

[13] Clifton CE, Morrow G. The kinetics of lysis of Escherichia coli. Journal of Bacteriology. 1936;31(5):441-451.

[14] Welcher FJ. The Analytical Uses of EDTA. New York, NY: D. Van Nostrand; 1965.

[15] Vogel AI. A Text Book of Quantitative Analysis. London, UK: Green; 1961.

[16] Geary WJ. The use of conductivity measurements in organic solvents for the characterisation of coordination compounds. Coordination Chemistry Reviews. 1971;7(1):81-122.

[17] Sreekanth A, Kurup MRP. Structural and spectral studies on four coordinate copper(II) complexes of 2-benzoylpyridine $N(4), N(4)$-(butane-1,4-diyl)thiosemicarbazone. Polyhedron. 2003;22(25-26):3321-3332.

[18] Cotton FA, Wilkinson G. Advanced Inorganic Chemistry. New Delhi, India: Wiley Eastern (Pvt); 1969.

[19] Padhyé SB, Kauffman GB. Transition metal complexes of semicarbazones and thiosemicarbazones. Coordination Chemistry Reviews. 1985;63:127-160.

[20] Thomas J, Parameswaran G. Structural, thermoanalytical and antitumour studies of metal chelates of anthracene-9carboxaldehyde thiosemicarbazone. Asian Journal of Chemistry. 2002;14(3):1354-1364.

[21] Murthy N, Dharmarajan TS. Synthesis, characterization and biological activity of copper(II) complexes with phenylglyoxal bis-(thiosemicarbazones). Asian Journal of Chemistry. 2002;14(3):1325-1330.

[22] Taylor MR, Glusker JP, Grabe EJ, Minkin JA. The crystal structure of the antitumor agent 3-ethoxy-2-oxobutyraldehyde bis(thiosemicarbazonato) copper(II). Bioinorganic Chemistry. 1974;3(3):189-205.

[23] Bellaci Ferrari M, Gasparri Fava G, Leporati E, et al. Synthesis, characterisation and biological activity of three copper(II) complexes with a modified nitrogenous base: 5formyluracil thiosemicarbazone. Journal of Inorganic Biochemistry. 1998;70(2):145-154.

[24] Garg Y, Samota MK, Seth G. Synthesis and antifungal activity of some metal complexes of 2-(2' hydroxybenzylidene)amino phenyl benzimidazole. Asian Journal of Chemistry. 2005;17(1):615-617.

[25] Singh BK. Complexation behaviour of glutathione with metal ions: a review. Asian Journal of Chemistry. 2005;17(1):1-32.

[26] Srivastava HP, Srivastava RK. Physicochemical studies of some 3d-bivalent transition metal complexes of glutathione(reduced). Journal of the Indian Chemical Society. 1995;72: $435-437$. 
[27] Tran-Ho L-C, May PM, Hefter GT. Complexation of copper(I) by thioamino acids. Implications for copper speciation in blood plasma. Journal of Inorganic Biochemistry. 1997; 68(3):225-231.

[28] Presta A, Stillman MJ. Chiral copper(I) -thiolate clusters in metallothionein and glutathione. Chirality. 1994;6(7):521530.

[29] Brouwer M, Brouwer TH. Biochemical defense mechanisms against copper-induced oxidative damage in the blue crab, Callinectes sapidus. Archives of Biochemistry and Biophysics. 1998;351(2):257-264.

[30] Radhakrishnan PS, Indrasenan P, Nair CGR. Complexes of lanthanide nitrates with $4 \mathrm{n}$-(2'-hydroxy benzylidene)aminoantipyrine. Polyhedron. 1984;3(1):67-70.

[31] Radhakrishnan PS, Indrasenan P. Indian Journal of Chemistry. 1989;28A:234.

[32] Yamguchi A, Penland RB, Mizushima S, Lane TJ, Curran C, Quagliano JV. Infrared absorption spectra of inorganic coordination compounds XIV. Infrared studies of some metalthiourea complexes. Journal of the American Chemical Society. 1958;80:527-529.

[33] Swaminathan K, Irving HMNH. Infra-red absorption spectra of complexes of thiourea. Journal of Inorganic and Nuclear Chemistry. 1964;26(7):1291-1294.

[34] Agarwal RK, Goel N, Sharma AK. Synthesis of some lanthanide(III) complexes derived from 4-[N-(4-methoxybenzyliene)amino] antipyrinethiosemicarbazone. Journal of the Indian Chemical Society. 2001;78:39-40.

[35] Rana VB, Jain PC, Swami MP, Srivastava AK. Thiosemicarbazones as ligands-I. Spectrochemical studies on trivalent metal complexes of 1-phenyl-orthohydroxy-4-benzamido thiosemicarbazone. Journal of Inorganic and Nuclear Chemistry. 1975;37(7-8):1826-1828.

[36] Stewart JE. Infrared absorption spectra of urea, thiourea, and some thiourea-alkali halide complexes. The Journal of Chemical Physics. 1957;26(2):248-254.

[37] Sadler PW. Hydrogen bonding in some thiosemicarbazones and thioamides. Journal of the Chemical Society (Resumed). 1961:957-960.

[38] Sarma BD, Bailer JC Jr. The stereochemistry of metal chelates with polydentate ligands. Part I. Journal of the American Chemical Society. 1955;77(21):5476-5480.

[39] West DX, Padhye SB, Sonawane PB, Chikte RC. Copper(II) complexes of tridentate (ONS) thiosemicarbazones. Asian Journal of Chemistry Reviews. 1990;1:125-137.

[40] Singh B, Mishra H. Complexes of cobalt(II), nickel(II), copper(II), zinc(II) cadmium(II) and dioxouranium(VI) with thiophene-2-aldehydethiosemicarbazones. Journal of the Indian Chemical Society. 1986;63:692-694.

[41] Singh B, Mishra H. Cobalt(II), nickel(II), copper(II), zinc(II) cadmium(II) and dioxouranium(VI) complexes with thiophene-2-aldehyde-4-phenylthiosemicarbazones. Journal of the Indian Chemical Society. 1986;63:1069-1070.

[42] Nakamoto K. Infrared Spectra of Inorganic and Coordination Compounds. New York, NY: John Wiley \& Sons; 1970.

[43] Bailey RA, Kozak SL, Michelson TW, Mills WN. Infrared spectra of complexes of the thiocyanate and related ions. Coordination Chemistry Reviews. 1971;6(4):407-445.

[44] Burmeister JL. Recent developments in the coordination chemistry of ambidentate ligands. Coordination Chemistry Reviews. 1966;1(1-2):205-221.
[45] Hester RE, Grossman WEL. Vibrational analysis of bidentate nitrate and carbonate complexes. Inorganic Chemistry. 1966;5:1308-1312.

[46] Hester RE, Grossman WEL. Vibrational analysis of bidentate nitrate and carbonate complexes. Inorganic Chemistry. 1966; 5(8):1308-1312.

[47] Topping G. Infra-red assignments and force constants in metal-nitrato complexes. Spectrochimica Acta. 1956;21(10): $1743-1751$.

[48] Agarwal RK, Arora K, Priyanka, Chakravorti I. Some high coordination compounds of thorium(IV) and dioxouranium(VI) with Schiff bases derived from 4-aminoantipyrine. Polish Journal of Chemistry. 1993;67:1913-1923.

[49] Lever ABP, Ramaswamy BS. Pyrazine metal complexes. Part III: derivatives of nickel(II). Journal of the Chemical Society (Resumed). 1963:5042-5048.

[50] Lever ABP. Electronic spectra of some transition metal complexes. Derivation of Dq and B. 1968;45:711-713.

[51] Tomlinson AAG, Hathaway BJ, Billing DE, Nichollas D. The electronic properties and stereochemistry of the copper(II) ion. Part V: the tetra-ammine complexes. Journal of the Chemical Society A: Inorganic, Physical, Theoretical. 1969:65-71.

[52] Chatterjee P, Duggal HK, Agarwala BV, Dey AK. Bipositive et al complexes of novel Schiff bases derived from carbazides and 2-aminopyridine. Journal of the Indian Chemical Society. 1989;66:550-553.

[53] Aravindakshan KK. Synthesis \& structural studies of some transition metal complexes of piperonaldehyde thiosemicarbazone. Indian Journal of Chemistry. 1987;26A:241-244.

[54] Mary NL, Parmeswaran G. Kinetics and mechanism of the thermal decomposition of Schiff base complexes of cobalt and nickel by TG and DSC studies. Asian Journal of Chemistry. 1993;5:597-605. 


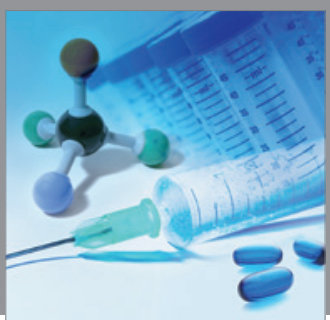

International Journal of

Medicinal Chemistry

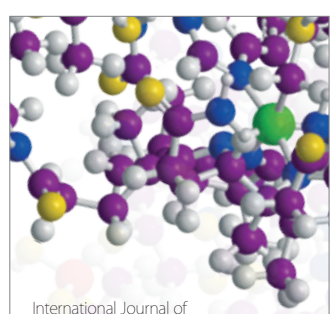

Carbohydrate Chemistry

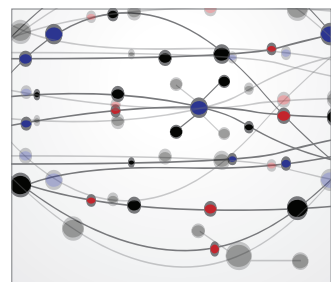

The Scientific World Journal
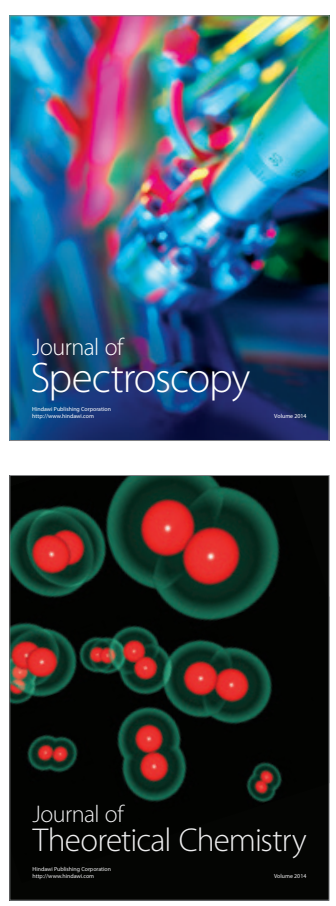
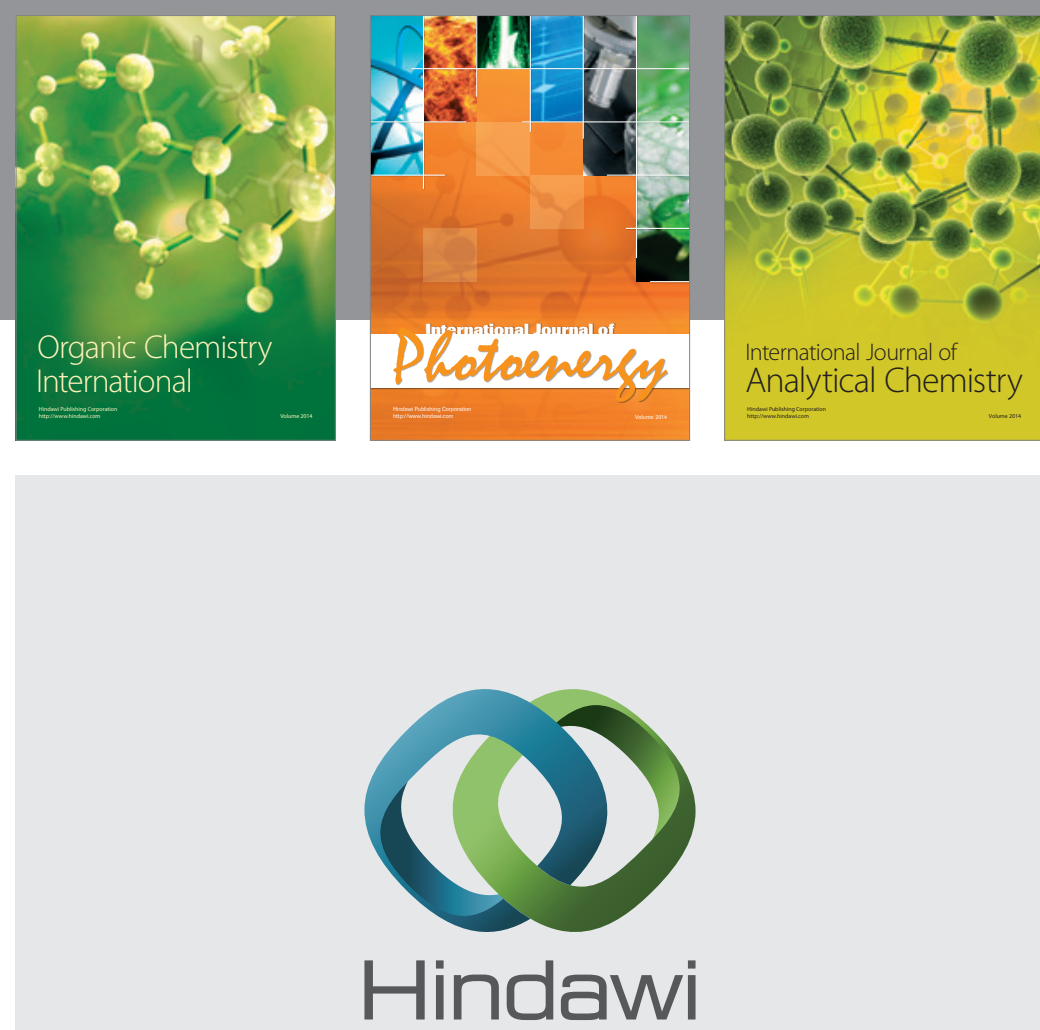

Submit your manuscripts at

http://www.hindawi.com
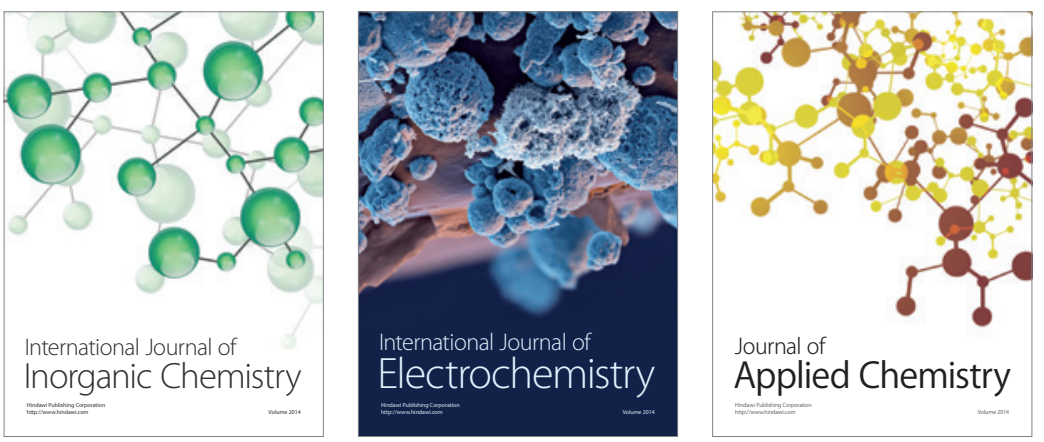

Journal of

Applied Chemistry
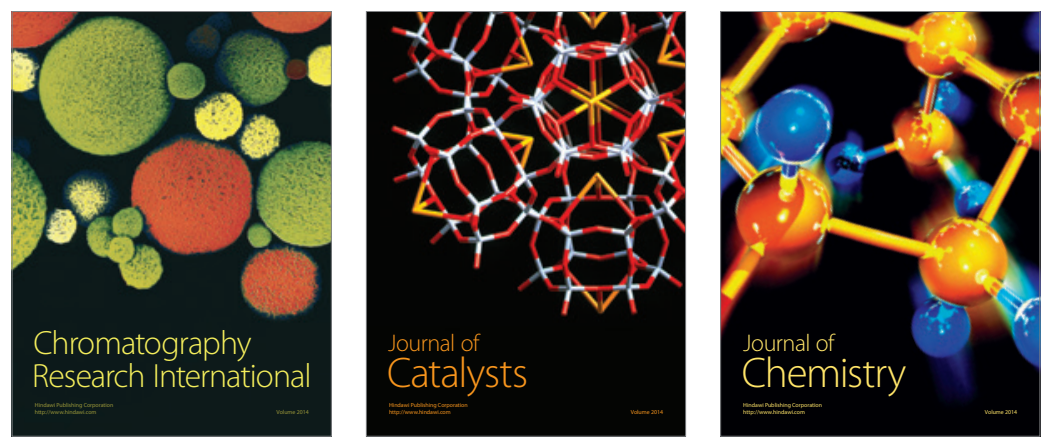
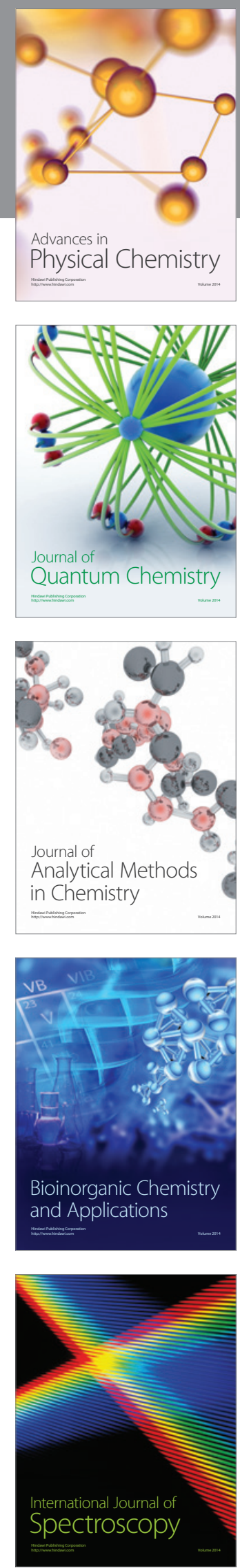